\title{
The Asymptotics of Recovery Probability in the Dual Renewal Risk Model with Constant Interest and Debit Force
}

\author{
Hao Wang and Lin Xu \\ Department of Statistics, Anhui Normal University, Wuhu, Anhui 241002, China \\ Correspondence should be addressed to Lin Xu; xulinahnu@gmail.com
}

Received 4 January 2015; Accepted 10 March 2015

Academic Editor: Alberto De Sole

Copyright (C) 2015 H. Wang and L. Xu. This is an open access article distributed under the Creative Commons Attribution License, which permits unrestricted use, distribution, and reproduction in any medium, provided the original work is properly cited.

The asymptotic behavior of the recovery probability for the dual renewal risk model with constant interest and debit force is studied. By means the idea of Markov Skeleton method, we studied the times that the random premium incomes happened and transformed the continuous time model into a discrete time model. By investigating the fluctuations of this discrete time model, we obtained the asymptotic behavior when the random premium income belongs to a kind of heavy-tailed distributions.

\section{Introduction}

The classical risk model is specified as

$$
U(t)=x+c t-S(t)
$$

where $x \geq 0$ is the initial surplus and $c$ is the constant rate at which the premiums are received. The aggregate claims process $\{S(t)\}$ is assumed to be a compound Poisson process, which denotes the total number of claims up to time $t$. Denote the time of arrival of the $i_{\text {th }}$ claim by $T_{i}$ and the size of the $i_{\text {th }}$ claim by $Y_{i}$. More details about the surplus process can be found in Asmussen and Albrecher [1] and Rolski et al. [2]. As pointed out by Albrecher et al. [3], its dual process may also be relevant for companies whose inherent business involves a constant flow of expenses while revenues arrive occasionally due to some contingent events (e.g., discoveries and sales). For instance, pharmaceutical or petroleum companies are prime examples of companies for which it is reasonable to model their surplus process as

$$
U_{t}=x-c t+\sum_{i=1}^{N_{t}} X_{k} .
$$

Here, $x$ is again the initial surplus, but the constant $c$ is now the rate of expenses, assumed to be deterministic and fixed. The aggregate claims process $S(t)=\sum_{k=1}^{N_{t}} X_{k}$ is assumed to be a compound renewal process. The negative claim sequence
$\left\{X_{k}, k=1,2,3, \ldots\right\}$ is assumed to be a sequence of independent and identically distributed (i.i.d.) nonnegative random variables (r.v.s.) with common distribution function $F=1-$ $\bar{F}$; we said $\bar{F}$ is the tailed distribution. The interoccurrence times $\left\{\theta_{k}, k=1,2,3, \ldots\right\}$ form another sequence of i.i.d. positive random variables. $\left\{\theta_{k}, k=1,2,3, \ldots\right\}$ and $\left\{X_{k}, k=\right.$ $1,2,3, \ldots\}$ are mutually independent. The occurrence times of the successive claims, $T_{n}=\sum_{k=1}^{n} \theta_{k}, n=1,2,3, \ldots$, constitute a renewal counting process

$$
N_{t}=\sharp\left\{n=1,2,3, \ldots: T_{n} \leq t\right\}, \quad t \geq 0 .
$$

The past decade has witnessed an increasing attention on the research of dual risk model. For example, see Albrecher et al. [3] for optimal dividend problem, see Cheung and Drekic [4] for dividend approximation and dual risk model with perturbation, and see Yao et al. [5] for optimal dividend and equity issuance. Due to the positive safety loading, in dual risk model, when the operation time scale is infinite, the ruin probability of dual risk model is zero; thus, it is meaningless to discuss the ruin problem under dual risk model. However, there is a constant consumption in the dual risk model; thus, it has occasionally happened that the surplus of dual risk model is negative; in this case, the decision maker will care the probability that the surplus of the model became positive; we define this probability as the "recovery probability." This conception is of both theoretical value and practical relevance. In this paper, we focus on the asymptotic behavior of the recovery probability under the dual renewal 
risk model, which covers the compound Poisson dual risk model. The rest of this paper is organized as follows. In Section 2, we present an introduction to the dual renewal risk model with constant interest force and debit interest force and the problem to be investigated. Section 3 provides the main results and the corresponding proofs.

\section{Model and Problem}

In this section, we consider the case that the research institution would like to invest his surplus in bond market or borrow money from the bank; both the investment interest force or the debit interest force are the same, say $\delta>0$. If there are no claims in the interval $(0, \Delta t)$, then the surplus up to time $t$ is given by

$$
U(t+\Delta t)=U(t)-c \Delta t+U(t)\left(e^{\delta \Delta t}-1\right) ;
$$

letting $\Delta t \rightarrow 0$, we can obtain

$$
U^{\prime}(t)=-c+\delta U(t) .
$$

It is easy to see, if $U(t)>c / \delta$, then $U^{\prime}(t)>0$; the surplus is increased and is not less than $c / \delta$; if $0<U(t)<c / \delta$, then $U^{\prime}(t)<0$; the surplus is decreased and the insurance company may be ruin; if $U(t)<0$, we know it is possible that the surplus $U(t)$ can recover to $c / \delta$. Above all, we can define the absolute positive profit as

$$
U(t)>\frac{c}{\delta} .
$$

In this paper, we will discuss the recovery probability which is the probability of the surplus recover to $c / \delta$ when $U(t) \leq 0$.

Now we let $\delta>0$ be the constant force of interest so that after time $t$ a capital $x$ becomes $x e^{\delta t}$. Then, the total surplus which is denoted by $W_{\delta}(t)$ is given by

$$
W_{\delta}(t)=x e^{\delta t}-c \int_{0}^{t} e^{\delta(t-y)}+\sum_{k=1}^{N_{t}} X_{k} e^{\delta\left(t-T_{k}\right)}, \quad t \geq 0 .
$$

Now, we can define the finite-time recovery probability as

$$
\psi(x, t)=P\left(\sup _{0 \leq s \leq t} W_{\delta}(s)>\frac{c}{\delta} \mid W_{\delta}(0)=x\right), \quad x \leq 0, t \geq 0 .
$$

At occurrence time $T_{n}=\sum_{k=1}^{n} \theta_{k}$, we observe the value $W_{\delta}\left(T_{n}\right)$ which represents the surplus immediately after paying the $n$th claim, $n=1,2, \ldots$ By virtue of (7), we have

$$
\begin{aligned}
W_{\delta}\left(T_{n}\right)= & x e^{\delta T_{n}}-c \int_{0}^{T_{n}} e^{\delta\left(T_{n}-y\right)} d y+\sum_{k=1}^{n} X_{k} e^{\delta\left(T_{n}-T_{y}\right)} \\
= & x e^{\delta T_{n-1}} \cdot e^{\delta \theta_{n}}-c \int_{0}^{T_{n-1}} e^{\delta\left(T_{n}-y\right)} d y \\
& -c \int_{T_{n-1}}^{T_{n}} e^{\delta\left(T_{n}-y\right)} d y+\sum_{k=1}^{n-1} X_{k} e^{\delta\left(T_{n}-T_{y}\right)}+X_{n} \\
= & W_{\delta}\left(T_{n-1}\right) e^{\delta \theta_{n}}-\frac{c}{\delta}\left(e^{\delta \theta_{n}}-1\right)+X_{n} .
\end{aligned}
$$

Let

$$
V_{n}=W_{\delta}\left(T_{n}\right)-\frac{c}{\delta}, \quad n=1,2, \ldots ;
$$

it follows that

$$
\begin{gathered}
V_{0}=W_{\delta}\left(T_{0}\right)-\frac{c}{\delta}=x-\frac{c}{\delta}, \\
V_{n}=V_{n-1} e^{\delta \theta_{n}}+X_{n}, \quad n=1,2, \ldots, \\
V_{n}=\left(x-\frac{c}{\delta}\right) \prod_{k=1}^{n} e^{\delta \theta_{k}}+\sum_{k=1}^{n} X_{k} \prod_{i=k+1}^{n} e^{\delta \theta_{i}}, \quad n=1,2, \ldots
\end{gathered}
$$

Since recovery can happen only at the time of a claim occurrence, we rewrite the finite-time recovery probability in (8) as

$$
\begin{aligned}
\psi(x, t) & =P\left(\sup _{0 \leq s \leq t} W_{\delta}(s)>\frac{c}{\delta} \mid W_{\delta}(0)=x\right) \\
& =P\left(\sup _{1 \leq n \leq N_{t}} V_{n}>0 \mid V_{0}=x-\frac{c}{\delta}\right) \\
& =P\left(\sup _{1 \leq n \leq N_{t}}\left(\left(x-\frac{c}{\delta}\right)+\sum_{k=1}^{n} X_{k} \prod_{i=1}^{k} Y_{i}\right)>0\right) .
\end{aligned}
$$

Since $\sup _{1 \leq n \leq N_{t}}\left\{\sum_{k=1}^{n} X_{k} \prod_{i=1}^{k} Y_{i}\right\}$ is increased, let $Y_{i}=e^{-\delta \theta_{i}}$; we obtain

$$
\psi(x, t)=P\left(\sum_{k=1}^{N_{t}} X_{k} \prod_{i=1}^{k} Y_{i}>\frac{c}{\delta}-x\right), \quad x \leq 0, t \geq 0 ;
$$

here, $\prod_{i=0}^{k} Y_{i}=e^{-\delta T_{k}} \in[0,1]$. So we can study the recovery probability in (8) by the (13).

Similarly, for the infinite-time recovery probability defined in (13), we have

$$
\psi(x, \infty)=P\left(\sum_{k=1}^{\infty} X_{k} \prod_{i=1}^{k} Y_{i}>\frac{c}{\delta}-x\right), \quad x \leq 0, t \geq 0 .
$$

Here and henceforth, all limit relationships are for $x \rightarrow \infty$ unless stated otherwise.

For two positive functions $f(x)$ and $g(x)$, the relation $f(x) \sim g(x)$ amounts to the conjunction of the relations $\limsup f(x) / g(x) \leq 1$ and $\lim \inf f(x) / g(x) \geq 1$, which are denoted as $f(x) \lesssim g(x)$ and $f(x) \gtrsim g(x)$, respectively. Next, we will define the convolution. For two distributions $F_{1}$ and $F_{2}$ on $[0, \infty)$, we can say $F_{1} * F_{2}$ is their convolution; that is,

$$
F_{1} * F_{2}(x)=\int_{0-}^{x} F_{1}(x-y) F_{2} d y, \quad x \geq 0 .
$$

Furthermore, we write $F^{1 *}=F$ and $F^{n *}=F^{(n-1) *} * F$ for every $n=2,3, \ldots$.

For the general renewal risk model, an asymptotic expression for the finite time ruin probability is presented in [6]; that is,

$$
\psi(x, t) \sim \lambda \exp \left\{\frac{\lambda}{\delta} \int_{\gamma e^{-\delta t}}^{\gamma} \frac{\mathbb{E} e^{s X}-1}{s} d s-\frac{\gamma c}{\delta}\right\} \int_{0}^{t} \bar{F}\left(x e^{\delta s}\right) d s .
$$


In recent years, with the study of classical model and the development of the financial and insurance business, more and more people are interested in dual risk model; however, an analogue problem in insurance company is the dividend payment or high gain tax payment. Thus, the problem studied here sounds reasonable.

\section{Main Results}

In order to complete our results, we should have some definitions and lemmas.

Definition 1. A distribution $F$ on $[0, \infty)$ is said to belong to the class $\mathfrak{F}$ if

$$
\lim _{x \rightarrow \infty} \frac{\overline{F^{n *}}(x)}{\bar{F}(x)}=n,
$$

for every $n \geq 2$; that is, $F \in \mathfrak{J}$.

Definition 2. A distribution $F$ on $[0, \infty)$ is said to belong to the class $\delta(\gamma)$ for some $\gamma \geq 0$ if

$$
\lim _{x \rightarrow \infty} \frac{\bar{F}(x-y)}{\bar{F}(x)}=e^{\gamma y},
$$

for every real number $y$ and the limit

$$
\lim _{x \rightarrow \infty} \frac{\overline{F^{2 *}}(x)}{\bar{F}(x)}=2 \int_{0-}^{\infty} e^{\gamma y} F(d y)
$$

exists and is finite; that is, $F \in S(\gamma)$. A larger class, $\mathscr{L}(\gamma)$, is defined by relation (18) alone.

A distribution function $F$ concentrated on $(-\infty, \infty)$ is still said to be subexponential to the right if $F^{+}(x)=$ $F(x) 1_{(0 \leq x<\infty)}$ is subexponential, and we usually denote by $\mathcal{S}$ the subexponential classes; see for example, [7]. Since it was introduced by [8-10], the subexponential class $\mathcal{S}$ has been extensively investigated by many researchers and applied to various fields. This class is often used to model claim-size distributions; see, for example, [11-13].

Definition 3. A distribution $F$ on $[0, \infty)$ is said to belong to $\mathscr{R}_{-\infty}$ if

$$
\lim _{x \rightarrow \infty} \frac{\bar{F}(x y)}{\bar{F}(x)}=0
$$

for arbitrary $y>1$.

Lemma 4. Let $F, F_{1}$, and $F_{2}$ be three distributions on $[0, \infty)$ such that $F \in S(\gamma)$ and that the $\lim l_{i}=\lim _{x \rightarrow \infty}\left(\overline{F_{i}}(x) / \bar{F}(x)\right)$ exists and is finite for $i=1,2$. Then,

$$
\lim _{x \rightarrow \infty} \frac{\overline{F_{1} * F_{2}}(x)}{\bar{F}(x)}=l_{1} \int_{0-}^{\infty} e^{\gamma y} F_{2}(d y)+l_{2} \int_{0-}^{\infty} e^{\gamma y} F_{1}(d y) .
$$

Lemma 5. Let $F_{1}$ and $F_{2}$ be two distributions on $[0, \infty)$. If $F_{1} \in$ $\delta(\gamma), F_{2} \in \mathscr{L}(\gamma)$, and $\overline{F_{2}}(x)=\circ\left(\overline{F_{1}}(x)\right)$, then $F_{1} * F_{2} \in \delta(\gamma)$ and

$$
\overline{F_{1} * F_{2}}(x) \sim \overline{F_{1}}(x) \int_{0-}^{\infty} e^{\gamma y} F_{2}(d y)+\overline{F_{2}}(x) \int_{0-}^{\infty} e^{\gamma y} F_{1}(d y) .
$$

Proof. See [6], Corollary 1.

Lemma 6. Let $F$ be a distribution on $[0, \infty)$. If $F \in S(\gamma)$, then it holds for each fixed $n=1,2, \ldots$ that

$$
\overline{F^{n *}}(x) \sim n\left(\int_{0-}^{\infty} e^{\gamma y} F(d y)\right)^{n-1} \bar{F}(x) .
$$

Proof. See [10], page 665.

Lemma 7. Let $\left\{X_{k}, k=1,2,3, \ldots\right\}$ be a sequence of independent and identically distributed (i.i.d.) nonnegative random variables with common distribution function $F, F \in$ $\mathfrak{J} .\left\{\sigma_{k}, k=1,2, \ldots\right\}$ is a sequence of nonnegative random variables, $\left\{\sigma_{k}, k=1,2, \ldots\right\}$ and $\left\{X_{k}, k=1,2, \ldots\right\}$ are mutually independent, and if for some $0<a \leq b<\infty$ and all $1 \leq k \leq n$, satisfies

$$
P\left(a \leq \sigma_{k} \leq b\right)=1,
$$

then

$$
P\left(\sum_{k=1}^{n} \sigma_{k} X_{k}>x\right) \sim \sum_{k=1}^{n} P\left(\sigma_{k} X_{k}>x\right) .
$$

Proof. See [7], Proposition 5.1.

Lemma 8. Let $\left\{N_{t}, t \geq 0\right\}$ be a renewal process. The interoccurrence time $\left\{\theta_{k}, k \geq 1\right\}$ forms another sequence of i.i.d. positive random variable with common distribution function $H$; it is easy to see that $H_{n}$ which is the distribution of the $n$th claim occurrence time $T_{n}=\sum_{k=1}^{n} \theta_{k}$ is the convolution of $H$. If $m(t)=\mathbb{E} N_{t}$ is the renewal function, then we have

$$
m(t)=\sum_{n=1}^{\infty} H_{n}(t)
$$

Proof. See [15], page 49.

\subsection{Main Results and Proof}

Theorem 9. In the dual renewal risk model with constant force of interest $\delta>0$, the number of claims $\left\{N_{t}, t \geq 0\right\}$ is a renewal process, $m(t)$ is the renewal function, and the claim sizes $\left\{X_{k}, k \geq 1\right\}$ and $\left\{N_{t}, t \geq 1\right\}$ are mutually independent. If $F \in \mathfrak{J} \cap \mathcal{S}(\gamma)$, then

$$
\psi(x, t) \sim \int_{0}^{t} e^{-\gamma(c / \delta) e^{\delta y}} P\left(X e^{-\delta y}>-x\right) d m(y), \quad x \leq 0,
$$

for arbitrary $t>0$. 
Proof. Starting with (13) and conditioning on $N_{t}$, we have

$$
\begin{aligned}
\psi(x, t) & =P\left(\sum_{k=1}^{N_{t}} X_{k} \prod_{i=1}^{k} Y_{i}>\frac{c}{\delta}-x\right) \\
& =\sum_{n=1}^{\infty} P\left(\sum_{k=1}^{N_{t}} X_{k} \prod_{i=1}^{k} Y_{i}>\frac{c}{\delta}-x \mid N_{t}=n\right) P\left(N_{t}=n\right) .
\end{aligned}
$$

Since $\prod_{i=1}^{k} Y_{i}=e^{-\delta T_{k}} \in\left[e^{-\delta t}, 1\right]$ for every $1 \leq k \leq n$, by Lemma 7, we have

$$
\begin{aligned}
\psi(x, t) & \sim \sum_{n=1}^{\infty} \sum_{k=1}^{n} P\left(X_{k} e^{-\delta T_{k}}>\frac{c}{\delta}-x\right) P\left(N_{t}=n\right) \\
& =\sum_{n=1}^{\infty} \sum_{k=1}^{n} P\left(X_{k} e^{-\delta T_{k}}>\frac{c}{\delta}-x, N_{t}=n\right) .
\end{aligned}
$$

We know $\left\{N_{t} \geq n\right\} \Leftrightarrow T_{n} \leq t$; then,

$$
\begin{aligned}
\psi(x, t) & \sim \sum_{k=1}^{\infty} \sum_{n=k}^{n} P\left(X_{k} e^{-\delta T_{k}}>\frac{c}{\delta}-x, N_{t}=n\right) \\
& =\sum_{k=1}^{\infty} P\left(X e^{-\delta T_{k}}>\frac{c}{\delta}-x, N_{t} \geq k\right) \\
& =\sum_{k=1}^{\infty} P\left(X e^{-\delta T_{k}}>\frac{c}{\delta}-x, T_{k}<t\right) \\
& =\sum_{k=1}^{\infty} \int_{0}^{t} P\left(X e^{-\delta y}>\frac{c}{\delta}-x\right) d H_{k}(y) \\
& =\int_{0}^{t} P\left(X e^{-\delta y}>\frac{c}{\delta}-x\right) d\left(\sum_{k=1}^{\infty} H_{k}(y)\right) \\
& =\int_{0}^{t} P\left(X e^{-\delta y}>\frac{c}{\delta}-x\right) d m(y) ;
\end{aligned}
$$

since $F \in \mathcal{S}(\gamma)$, then

$$
\frac{\lim _{x \rightarrow \infty} P\left(X>(c / \delta) e^{\delta y}-x e^{\delta y}\right)}{P\left(X>-x e^{\delta y}\right)}=e^{-\gamma(c / \delta) e^{\delta y}} .
$$

So we have

$$
\psi(x, t) \sim \int_{0}^{t} e^{-\gamma(c / \delta) e^{\delta y}} P\left(X e^{-\delta y}>-x\right) d m(y) .
$$

We present an asymptotic expression for the finite-time recovery probability in Theorem 9 . In the following, we will show the last main result of the paper.
Theorem 10. In the dual renewal risk model with constant force of interest $\delta>0$, if $F \in \mathcal{S} \cap \mathscr{R}_{-\infty}$ for some $\gamma \geq 0, x \leq 0$, then

$$
\begin{gathered}
\mathbb{E} e^{\gamma S_{\infty}}<\infty, \quad \text { where } S_{\infty}=\sum_{k=1}^{\infty} X_{k} \prod_{i=1}^{k} Y_{i}, \\
\psi(x, \infty) \sim \mathbb{E} e^{\gamma S_{\infty}} P\left(X_{1} Y_{1}>\frac{c}{\delta}-x\right), \quad x \leq 0,
\end{gathered}
$$

where $\left\{Y_{i}=e^{-\delta \theta_{i}}, i=1,2, \ldots\right\}$ is a sequence of i.i.d. positive random variables with common distribution function $G$.

Proof. The proof of $\mathbb{E} e^{\gamma S_{\infty}}<\infty$ is the same as the one for Theorem 3.2 of [16]; we do not copy the steps here. Next, we will prove (34). Let $\widetilde{S}_{\infty}$ be a copy of $S_{\infty}$ independent of $\left\{\left(X_{k}, Y_{k}\right), k=1,2, \ldots\right\}$. Then, for every $n=1,2, \ldots$,

$$
S_{\infty} \triangleq S_{T_{n}}+\widetilde{S}_{\infty} \prod_{i=1}^{n} Y_{i}
$$

Therefore,

$$
S_{\infty} \geq S_{T_{n}}
$$

$$
S_{\infty} \leq S_{T_{n}}+X^{*} \prod_{i=1}^{n} Y_{i}
$$

here $X^{*}=\left(Z \mid Z>c / \delta-x_{0}\right)$ is a new conditional random variable, and the distribution still belongs to the intersection $\mathcal{S}(\gamma) \cap \mathscr{R}_{-\infty} ; x_{0}<0$ is a small enough constant.

So from (36) and according to the (13), we can obtain

$$
\begin{aligned}
P( & \left.S_{\infty}>\frac{c}{\delta}-x\right) \\
& \geq P\left(S_{T_{n}}>\frac{c}{\delta}-x\right) \\
& =P\left(\sum_{k=1}^{N_{t}} X_{k} \prod_{i=1}^{k} Y_{i}>\frac{c}{\delta}-x\right) \\
& =P\left(X_{1} Y_{1}+\sum_{k=2}^{N_{t}} X_{k} \prod_{i=2}^{k} Y_{i}>\frac{c}{\delta}-x\right) \\
& =P\left(\left(X_{1}+\sum_{k=2}^{N_{t}} X_{k} \prod_{i=2}^{k} Y_{i}\right) Y_{1}>\frac{c}{\delta}-x\right) \\
& =\int_{0}^{1} P\left(X_{1}+\sum_{k=2}^{N_{t}} X_{k} \prod_{i=2}^{k} Y_{i}>\frac{(c / \delta-x)}{y}\right) G(d y) .
\end{aligned}
$$

Since

$$
\begin{aligned}
& P\left(\sum_{k=2}^{N_{t}} X_{k} \prod_{i=2}^{k} Y_{i}>\frac{c}{\delta}-x\right) \\
& \leq P\left(\sum_{k=2}^{N_{t}} X_{k} Y_{1}>\frac{c}{\delta}-x\right) \\
& =\int_{0}^{1} P\left(\sum_{k=2}^{N_{t}} X_{k}>\frac{(c / \delta-x)}{y}\right) G(d y)
\end{aligned}
$$


and the Lemma 6, we can see

$$
\begin{aligned}
& P\left(\sum_{k=2}^{N_{t}} X_{k}>\frac{(c / \delta-x)}{y}\right) \\
& \sim\left(N_{t}-1\right)\left(\int_{0-}^{\infty} e^{\gamma y} F(d y)\right)^{N_{t}-2} \bar{F}\left(\frac{(c / \delta-x)}{y}\right) \\
& \quad=\left(N_{t}-1\right)\left(\mathbb{E} e^{\gamma X}\right)^{N_{t}-2} \bar{F}\left(\frac{(c / \delta-x)}{y}\right) .
\end{aligned}
$$

We know $F \in \mathscr{R}_{-\infty}$, so $\lim _{x \rightarrow \infty}(\bar{F}(x y) / \bar{F}(x))=0, y>1$, that is, $\bar{F}(x y)=\circ(\bar{F}(x))$, we can obtain $\bar{F}(-x / y)=\circ(\bar{F}(-x))$. So

$$
\begin{aligned}
& P\left(\sum_{k=2}^{N_{t}} X_{k} \prod_{i=2}^{k} Y_{i}>\frac{c}{\delta}-x\right) \\
& \quad \leq\left(N_{t}-1\right)\left(\mathbb{E} e^{\gamma X}\right)^{N_{t}-2} \bar{F}\left(\frac{(c / \delta-x)}{y}\right) \\
& \quad=\circ\left(\bar{F}\left(\frac{c}{\delta}-x\right)\right) ;
\end{aligned}
$$

we deduce that

$$
\begin{gathered}
\frac{\lim _{x \rightarrow \infty} P\left(\sum_{k=2}^{N_{t}} X_{k} \prod_{i=2}^{k} Y_{i}>c / \delta-x\right)}{\bar{F}(c / \delta-x)}=0, \\
\frac{\lim _{x \rightarrow \infty} P\left(X_{1}>c / \delta-x\right)}{\bar{F}(c / \delta-x)}=1 .
\end{gathered}
$$

By Lemma 4, we obtain that

$$
\begin{aligned}
P( & \left.X_{1}+\sum_{k=2}^{N_{t}} X_{k} \prod_{i=2}^{k} Y_{i}>\frac{(c / \delta-x)}{y}\right) \\
\sim & \left(1 \cdot \int_{0-}^{\infty} e^{\gamma y} F_{2}(d y)+0 \cdot \int_{0-}^{\infty} e^{\gamma y} F_{1}(d y)\right) \\
& \cdot \bar{F}\left(\frac{(c / \delta-x)}{y}\right) \\
= & \int_{0-}^{\infty} e^{\gamma y} F_{2}(d y) \bar{F}\left(\frac{(c / \delta-x)}{y}\right) \\
= & \mathbb{E} e^{\gamma \sum_{k=2}^{N_{t}} X_{k} \prod_{i=2}^{k} Y_{i}} \bar{F}\left(\frac{(c / \delta-x)}{y}\right),
\end{aligned}
$$

so

$$
\begin{aligned}
& P\left(S_{\infty}>\frac{c}{\delta}-x\right) \\
& \quad \geq \mathbb{E} e^{\gamma \sum_{k=2}^{N_{t}} X_{k} \prod_{i=2}^{K} Y_{i}} \int_{0}^{1} \bar{F}\left(\frac{(c / \delta-x)}{y}\right) G(d y) \\
& \quad=\mathbb{E} e^{\gamma \sum_{k=2}^{N_{t}} X_{k} \prod_{i=2}^{K} Y_{i}} P\left(X_{1} Y_{1}>\frac{c}{\delta}-x\right) .
\end{aligned}
$$

Then,

$$
\liminf _{x \rightarrow \infty} \frac{P\left(S_{\infty}>c / \delta-x\right)}{P\left(X_{1} Y_{1}>c / \delta-x\right)} \geq \mathbb{E} e^{\gamma\left(\sum_{k=2}^{N_{t}} X_{k} \prod_{i=2}^{k} Y_{i}\right)} .
$$

Clearly, $\sum_{k=2}^{N_{t}} X_{k} \prod_{i=2}^{k} Y_{i}$ converges to $S_{\infty}$ in distribution as $n \rightarrow \infty$. Therefore, by the dominated convergence theorem, the expectation on the right-hand side above converges to $\mathbb{E} e^{\gamma S_{\infty}}$ as $n \rightarrow \infty$; that is,

$$
\liminf _{x \rightarrow \infty} \frac{P\left(S_{\infty}>c / \delta-x\right)}{P\left(X_{1} Y_{1}>c / \delta-x\right)} \geq \mathbb{E} e^{\gamma S_{\infty}} .
$$

It is easy to construct the corresponding asymptotic upper bound by (37). Similarly as above (by Lemmas 4 and 5),

$$
\limsup _{x \rightarrow \infty} \frac{P\left(S_{\infty}>c / \delta-x\right)}{P\left(X_{1} Y_{1}>c / \delta-x\right)} \leq \mathbb{E} e^{\gamma\left(\sum_{k=2}^{N_{t}} X_{k} \prod_{i=2}^{k} Y_{i}+X^{*} \prod_{i=2}^{n} Y_{i}\right)} .
$$

Clearly, $\sum_{k=2}^{N_{t}} X_{k} \prod_{i=2}^{k} Y_{i}+X^{*} \prod_{i=2}^{n} Y_{i}$ converges to $S_{\infty}$ in distribution as $n \rightarrow \infty$. Therefore, similarly as above,

$$
\limsup _{x \rightarrow \infty} \frac{P\left(S_{\infty}>c / \delta-x\right)}{P\left(X_{1} Y_{1}>c / \delta-x\right)} \leq \mathbb{E} e^{\gamma S_{\infty}} .
$$

This completes the proof.

\section{Conflict of Interests}

The authors declare that there is no conflict of interests regarding the publication of this paper.

\section{References}

[1] S. Asmussen and H. Albrecher, Ruin Probabilities, vol. 14, World Scientific, Singapore, 2010.

[2] T. Rolski, H. Schmidli, V. Schmidt, and J. Teugels, Stochastic Processes for Insurance and Finance, John Wiley \& Sons, 2009.

[3] H. Albrecher, A. Badescu, and D. Landriault, "On the dual risk model with tax payments," Insurance: Mathematics and Economics, vol. 42, no. 3, pp. 1086-1094, 2008.

[4] E. C. K. Cheung and S. Drekic, "Dividend moments in the dual risk model: exact and approximate approaches," ASTIN Bulletin, vol. 38, no. 2, pp. 399-422, 2008.

[5] D. Yao, H. Yang, and R. Wang, "Optimal financing and dividend strategies in a dual model with proportional costs," Journal of Industrial and Management Optimization, vol. 6, no. 4, pp. 761777, 2010.

[6] D. B. H. Cline, "Convolution tails, product tails and domains of attraction," Probability Theory and Related Fields, vol. 72, no. 4, pp. 529-557, 1986.

[7] Q. Tang and G. Tsitsiashvili, "Randomly weighted sums of subexponential random variables with application to ruin theory," Extremes, vol. 6, no. 3, pp. 171-188, 2003.

[8] V. P. Chistyakov, "A theorem on sums of independent positive random variables and its applications to branching random processes," Theory of Probability and Its Applications, vol. 9, pp. 640-648, 1964.

[9] J. Chover, P. Ney, and S. Wainger, "Functions of probability measures," Journal d'Analyse Mathématique, vol. 26, no. 1, pp. 255-302, 1973.

[10] J. Chover, P. Ney, and S. Wainger, "Degeneracy properties of subcritical branching processes," The Annals of Probability, vol. 1, no. 4, pp. 663-673, 1973. 
[11] P. Embrechts and N. Veraverbeke, "Estimates for the probability of ruin with special emphasis on the possibility of large claims," Insurance Mathematics and Economics, vol. 1, no. 1, pp. 55-72, 1982.

[12] C. Klüppelberg, "Estimation of ruin probabilities by means of hazard rates," Insurance: Mathematics and Economics, vol. 8, no. 4, pp. 279-285, 1989.

[13] Q. Tang and G. Tsitsiashvili, "Finite- and infinite-time ruin probabilities in the presence of stochastic returns on investments," Advances in Applied Probability, vol. 36, no. 4, pp. 12781299, 2004.

[14] B. A. Rogozin and M. S. Sgibnev, "Banach algebras of measures on the real axis with the given asymptotics of distributions at infinity," Siberian Mathematical Journal, vol. 40, no. 3, pp. 565576, 1999.

[15] A. Gut, Stopped Random Walks, Springer, Berlin, Germany, 1988.

[16] D. G. Konstantinides, K. W. Ng, and Q. Tang, “The probabilities of absolute ruin in the renewal risk model with constant force of interest," Journal of Applied Probability, vol. 47, no. 2, pp. 323334, 2010. 


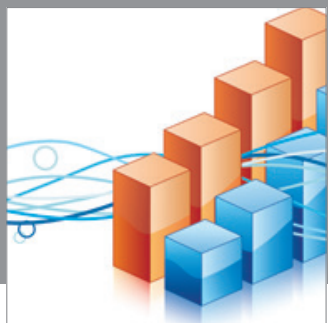

Advances in

Operations Research

mansans

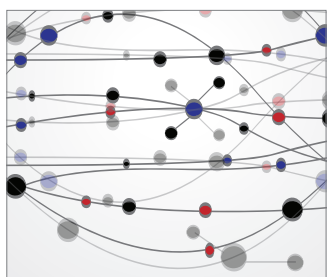

The Scientific World Journal
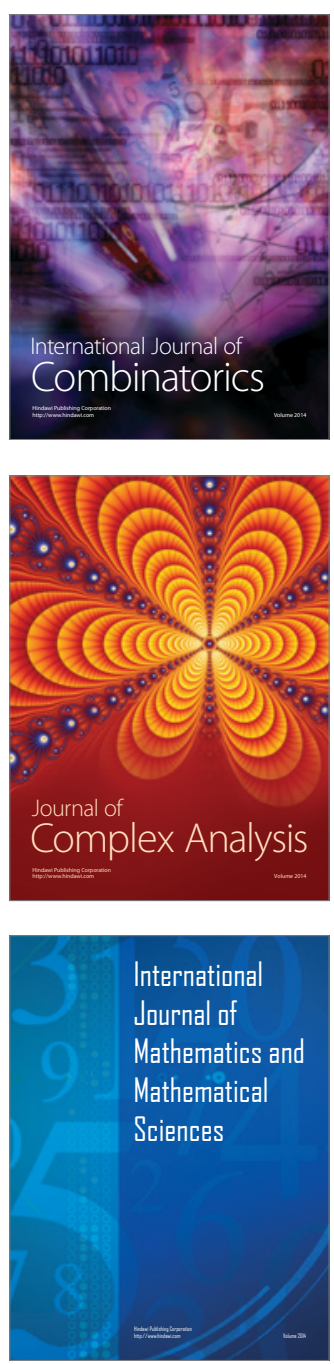
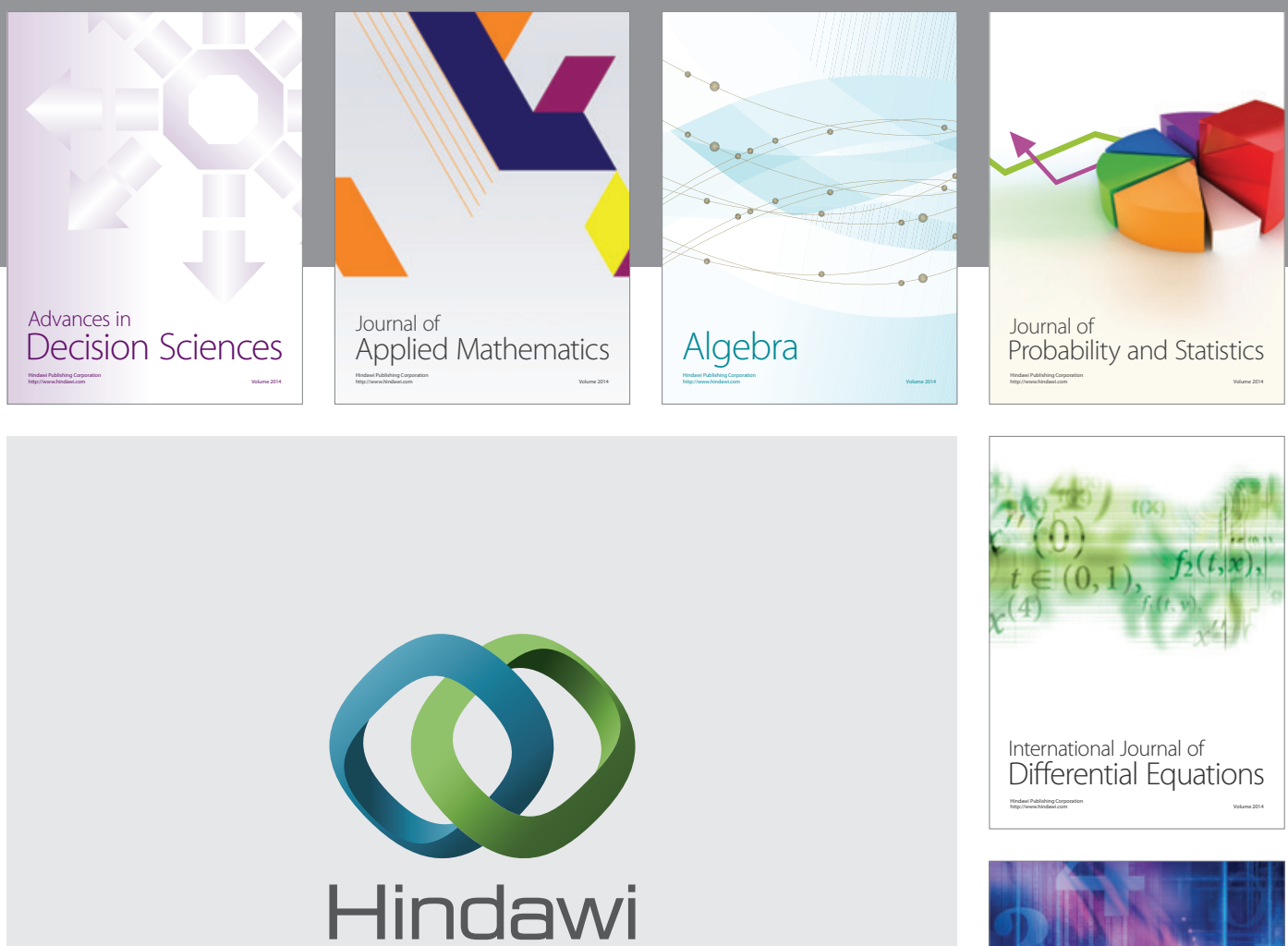

Submit your manuscripts at http://www.hindawi.com
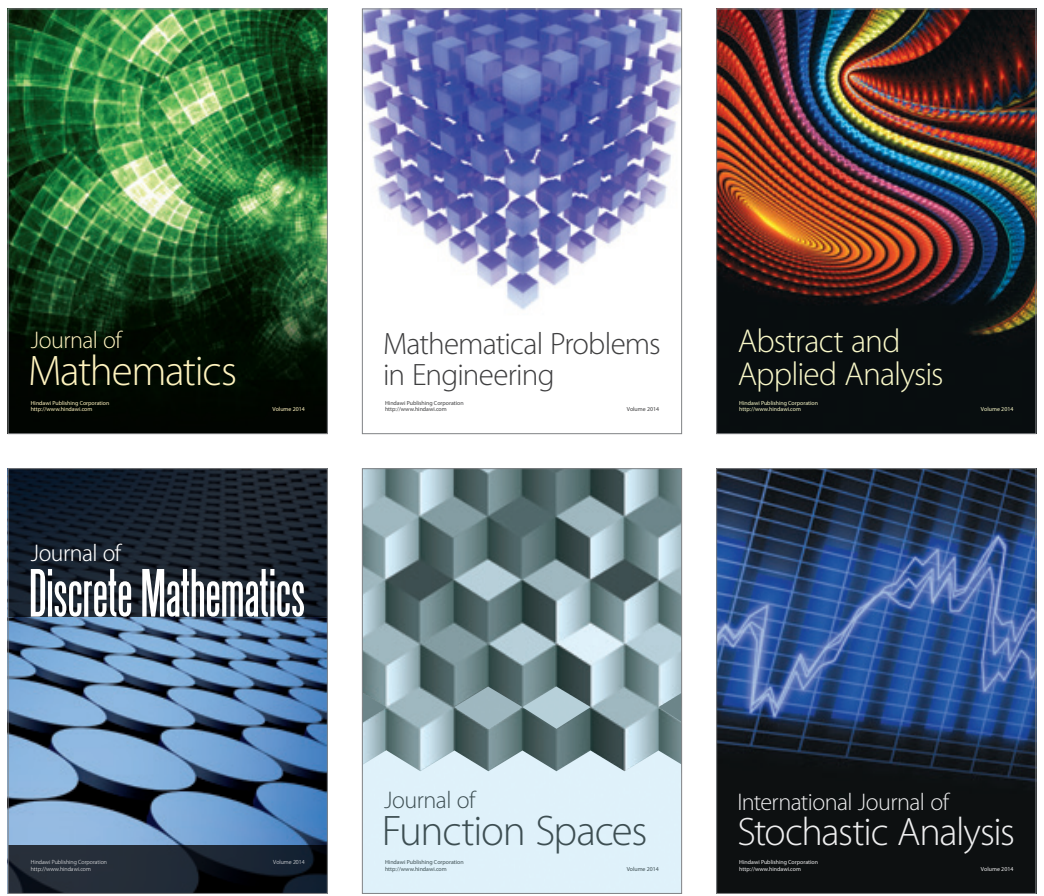

Journal of

Function Spaces

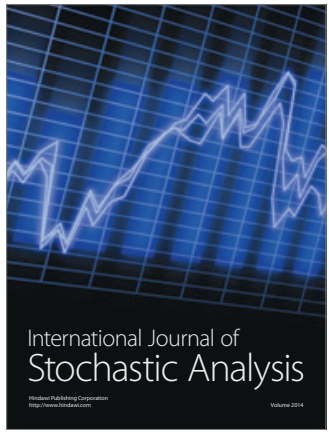

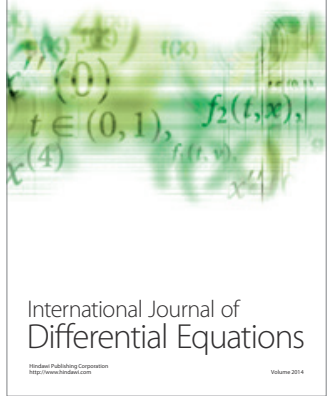
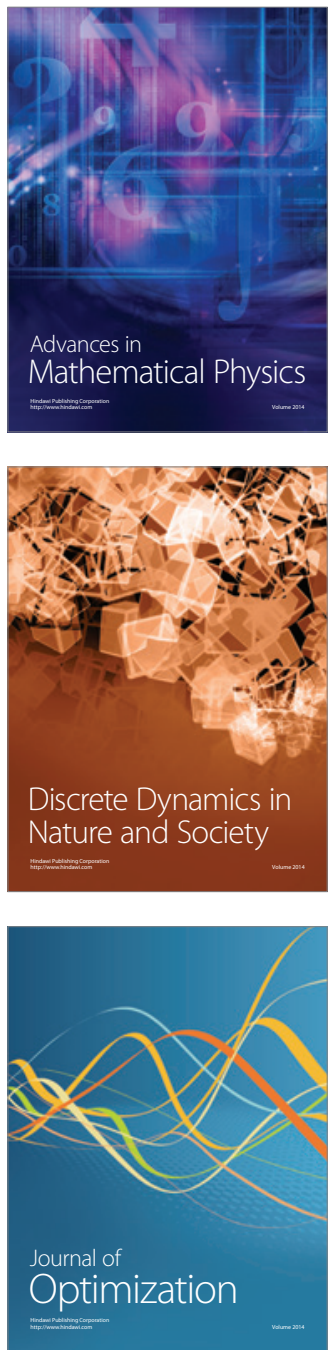